\title{
Characterization of two different types of UDP-glucose/-galactose 4-epimerase involved in galactosylation in fission yeast
}

\author{
Correspondence \\ Naotaka Tanaka \\ ntanaka@ag.kagawa-u.ac.jp
}

Received 4 October 2009

Revised 19 November 2009

Accepted 24 November 2009

\author{
Shotaro Suzuki, ${ }^{1}$ Tomohiko Matsuzawa, ${ }^{1}$ Yayoi Nukigi, ${ }^{1}$ Kaoru Takegawa ${ }^{2}$ \\ and Naotaka Tanaka ${ }^{1}$
}
${ }^{1}$ Department of Applied Biological Sciences, Faculty of Agriculture, Kagawa University, Miki-cho, Kagawa 761-0795, Japan
${ }^{2}$ Department of Bioscience and Biotechnology, Faculty of Agriculture, Kyushu University, 6-10-1 Hakozaki, Fukuoka 812-8581, Japan

\begin{abstract}
Schizosaccharomyces species are currently the only known organisms with two types of genes encoding UDP-glucose/-galactose 4-epimerase, uge $1^{+}$and gal10 ${ }^{+}$. A strain deleted for uge ${ }^{+}$ exhibited a severe galactosylation defect and a decrease in activity and in UDP-galactose content when grown in glucose-rich medium ( $2 \%$ glucose), indicating that Uge1p is a major UDPglucose/-galactose 4-epimerase under these growth conditions. In contrast, ga/10 ${ }^{+}$was efficiently expressed and involved in galactosylation of cell-surface proteins in low-glucose medium ( $0.1 \%$ glucose and $2 \%$ glycerol), but not in galactose-containing medium. In a uge $1 \Delta$ gal10 $\Delta$ strain, the galactosylation defect was suppressed and UDP-galactose content restored to wild-type levels in galactose-containing medium. Disruption of $\mathrm{ga} / 7^{+}$, encoding galactose-1-phosphate uridylyltransferase, in the uge $1 \Delta$ gal10 $\Delta$ strain reversed suppression of the galactosylation defect and reduced levels of UDP-galactose, indicating that galactose is transported from the medium to the cytosol and is converted into UDP-galactose via galactose 1-phosphate by Gal7p in Sch. pombe.
\end{abstract}

\section{INTRODUCTION}

Glycoproteins in the fission yeast Schizosaccharomyces pombe contain a large amount of galactose in addition to both $\mathrm{N}$ - and O-linked mannan (Manners \& Meyer, 1977; Moreno et al., 1985; Ikeda et al., 2009; Ohashi et al., 2009), indicating that Sch. pombe is equipped with mechanisms for glycoprotein galactosylation like animal cells. Research has demonstrated that the outer chain structure of galactomannan has an $\alpha$-1,2-linked galactose or pyruvylated galactose attached to a poly- $\alpha-1,6$-linked mannose backbone (Gemmill \& Trimble, 1998), and that the pyruvylated galactose epitope bears remarkable structural resemblance to the $\mathrm{N}$-acetylneuraminic acid-linked galactose epitope found in mammalian cells (Gemmill \& Trimble, 1996). These galactose residues are enzymically modified by galactosyltransferases using UDP-galactose as substrate (Andreishcheva et al., 2004; Chappell et al., 1994; Yoko-o et al., 1998). UDP-galactose is transported to the Golgi lumen from the cytosol by the Golgi-localized UDPgalactose transporter (Gmslp) (Tabuchi et al., 1997; Tanaka \& Takegawa, 2001). In Sch. pombe, the precise manner in which UDP-galactose is synthesized in the

Abbreviations: HRP-PNA, horseradish peroxidase-conjugated peanut lectin. cytosol has been unclear. In the cytosol, the key enzyme for UDP-galactose synthesis is UDP-galactose/-glucose 4epimerase, which catalyses the interconversion of UDPgalactose and UDP-D-glucose. Interestingly, Schizosaccharomyces species have two types of UDP-glucose/-galactose 4 -epimerase, while other organisms have only one. It is not known why two types of epimerase are needed and how they are regulated in Sch. pombe.

In Saccharomyces cerevisiae, Gal10p is the sole UDPglucose/-galactose 4-epimerase, which consists of a UDPglucose/-galactose 4-epimerase domain and a galactose mutarotase domain (Majumdar et al., 2004; Scott \& Timson, 2007; Thoden \& Holden, 2005), and which catalyses mutarotation of $\alpha$-galactose to $\beta$-galactose. Expression of the GAL10 gene is regulated by Gal4p, a DNA-binding transcription factor required for activation of the GAL genes in cells growing on galactose as sole carbon source (De Robichon-Szulmajster, 1958; Platt \& Reece, 1998; Lohr et al., 1995). In contrast to Sch. pombe, UDP-galactose is not used as a glycosylation substrate by glycosyltransferases because secreted glycoproteins are mannosylated in Sac. cerevisiae. Therefore, in Sac. cerevisiae, UDP-galactose and UDP-glucose/-galactose 4-epimerase function solely in galactose metabolism. 
The metabolism of galactose in most organisms occurs via the Leloir pathway (Frey, 1996; Holden et al., 2003), comprising galactokinase (EC 2.7.1.6), galactose-1-phosphate uridylyltransferase (EC 2.7.7.12) and UDP-glucose/galactose 4-epimerase (EC 5.1.3.2) (see Fig. 7b). In the first step of this pathway, cytosolic $\alpha$-D-galactose is phosphorylated by ATP-dependent galactokinase to yield galactose 1phosphate (Gal-1-P). Next, Gal-1-P uridylyltransferase transfers UMP from UDP-glucose (UDP-Glc) to Gal-1-P via a two-step ping-pong mechanism, releasing glucose 1phosphate (Glc-1P) and forming UDP-galactose. Finally, $\mathrm{NAD}^{+}$-dependent UDP-galactose-4-epimerase converts UDP-galactose to UDP-Glc. The glucose 1-phosphate is used in glycolysis and the UDP-galactose is utilized in mammals for galactosylation of glycoproteins.

In Sch. pombe, the metabolism of galactose is poorly understood because cells are not able to grow on galactose as a sole carbon source. This phenotype makes it difficult to analyse the Leloir pathway, despite the fact that genes encoding galactokinase, Gal-1-P uridylyltransferase and UDP-glucose/-galactose epimerase are present in the Sch. pombe genome.

In the present study, we describe the role of two types of UDP-galactose/-glucose 4-epimerase in Sch. pombe, Ugelp and Gal10p.

\section{METHODS}

Strains, medium and genetic methods. The wild-type $S c h$. pombe strain ARC039 ( $h^{-}$leu1-32 ura4-C190T) was used. The gms1s strain was constructed as described by Tabuchi et al. (1997). Standard rich medium (YES) and synthetic minimal medium (MM) for growing Sch. pombe were used as described by Moreno et al. (1991). Sch. pombe cells were transformed by the lithium acetate method or by electroporation (Morita \& Takegawa, 2004; Okazaki et al., 1990; Suga \& Hatakeyama, 2001; Suga et al., 2000). Escherichia coli XL1-blue (Stratagene) was used for all cloning procedures. Standard genetic methods have been described (Alfa et al., 1993).

Gene disruptions. The $u g e 1^{+}$locus (SPBC365.14) was disrupted in the wild-type $S c h$. pombe strain by replacing an internal $u g e 1^{+}$gene fragment with the Sch. pombe $\mathrm{ura4}^{+}$gene. To amplify the $u g e 1^{+}$gene by PCR, the following oligonucleotides were used: sense, $5^{\prime}$ GTAGTCGCATGAAGCTGGTGGTG-3' , and antisense, 5' -ACCTAATCGATAGCTCGAGCGC-3' ${ }^{\prime}$. A $1.97 \mathrm{kbp}$ fragment was recovered and ligated into pGEM-T EASY vector (Promega). A HindIII site within the cloned $\mathrm{ugel}^{+}$open reading frame was digested and a $1.6 \mathrm{kbp}$ $\mathrm{ura}^{+}$gene was inserted.To disrupt the gallo ${ }^{+}$locus (SPBPB2B2.12C), the following oligonucleotides were used: sense, 5'-CATCTGTGTCTTTATTCTTCTGTGCCCCC-3' , and antisense, 5'-GAACAAAAAGATGCTTAATCGGCTGGG-3'. A HindIII site within the cloned $g a l 10^{+}$open reading frame was digested and the $\mathrm{ura4}^{+}$gene was inserted.To disrupt the gal $^{+}$locus (SPBPB2B2.10c), the following oligonucleotides were used: sense, 5'-GTACGATCCAACATGTTACCTCTGCCCTGG-3', and antisense, 5'-GGAGCTTGGTGAAGACCCATTGAGTAGGGG-3'. A HindIII site within the cloned $\mathrm{gall}^{+}$open reading frame was digested and the $\mathrm{urat}^{+}$gene was inserted. Linearized DNA fragments carrying the disrupted uge $^{+},{\text {gal } 10^{+}}$and gal7 $^{+}$genes, respectively, were used to transform the wild-type haploid ARC039 strain, and $\mathrm{ura}^{+}{ }^{+}$transformants were selected. To confirm that the corresponding individual genes had been disrupted, $\mathrm{ura}^{+}$transformants were analysed by Southern blotting and PCR to verify correct integration of the deletion constructs.

Lectin staining. Horseradish peroxidase-conjugated peanut lectin (HRP-PNA) was used to detect galactose residues on the cell surface as described previously (Takegawa et al., 1996). Briefly, cells were grown to stationary phase in YES or MM at $30{ }^{\circ} \mathrm{C}$. Harvested cells were washed twice with distilled water and then resuspended in distilled water. Cells were fractured using glass beads to yield a crude protein extract. Extracted protein was spotted onto filter paper and dried. The filters were then washed twice in $10 \mathrm{mM}$ Tris/HCl buffer (pH 7.4), $0.15 \mathrm{M} \mathrm{NaCl}$ and $0.05 \%$ Tween 20 at room temperature for $5 \mathrm{~min}$, followed by addition of HRP-PNA at room temperature and incubation for $1 \mathrm{~h}$ with continuous shaking. The filter paper was incubated in $0.03 \% 3,3^{\prime}$-diaminobenzidine and $0.003 \% \mathrm{H}_{2} \mathrm{O}_{2}$ in PBS until spots were visible.

Acid phosphatase staining. Glycosylation of acid phosphatase was analysed as described by Huang \& Snider (1995), with minor modifications. Briefly, cells were grown in $5 \mathrm{ml} \mathrm{MM}$ medium to midexponential phase at $30^{\circ} \mathrm{C}$. To induce acid phosphatase, cells were centrifuged, washed and resuspended in $5 \mathrm{ml}$ phosphate-free $\mathrm{MM}$ medium, and incubated for $12 \mathrm{~h}$ at $30{ }^{\circ} \mathrm{C}$. Cells were then collected by centrifugation, washed once in $62.5 \mathrm{mM} \mathrm{Tris} / \mathrm{HCl}(\mathrm{pH} 6.8)$, and suspended in $240 \mu \mathrm{l}$ lysis buffer $(62.5 \mathrm{mM}$ Tris/ $\mathrm{HCl} \mathrm{pH} 6.8,1 \mathrm{mM}$ EDTA, $2 \mathrm{mM}$ phenylmethylsulfonyl fluoride, $0.1 \mathrm{mM}$ dithiothreitol and $10 \%$ glycerol, $\mathrm{pH} 6.8$ ). A cell lysate were prepared using $0.5 \mathrm{~mm}$ glass beads in a Mini BeadBeater (BioSpec Products). The lysate was recovered and centrifuged at $15000 \mathrm{~g}$ for $10 \mathrm{~min}$ at $4{ }^{\circ} \mathrm{C}$, and the supernatant was loaded onto a $6 \%$ native polyacrylamide gel. Electrophoresis and staining of acid phosphatase activity were performed as described by Schweingruber et al. (1986).

Northern-blot analysis. Total RNA from cultures with an $\mathrm{OD}_{600}$ of 0.8-1.0 was extracted by the glass bead method described above (Sambrook et al., 1989). An aliquot $(20 \mu \mathrm{g})$ of total RNA was separated on a $1 \%(\mathrm{w} / \mathrm{v})$ agarose gel containing $20 \%$ formaldehyde. Subsequently, RNA was blotted onto Hybond-N membranes in $10 \times$ SSC buffer (1.5 M NaCl, $0.15 \mathrm{M}$ sodium citrate, $\mathrm{pH} \mathrm{7.0)}$ and hybridized with gene-specific probes. The probes were obtained by PCR using the following primers. 5'-GGTTGTTACTTTGGTGAGAGCGCACTGAGGAC-3' and 5'-CAGCCTTAGTAATATCAGCGGTAGAAGCC-3' for $\mathrm{leu1}^{+}$(constitutive endogenous control), 5' CGGAGGTGCTCATCCCTCTGGTGAACTCGG-3' and $5^{\prime}$-TTACTTATATGTCTTGGTATGGGTCAGGTC- $3^{\prime}$ for $\mathrm{uge}^{+}$, and $5^{\prime}-\mathrm{AAT}-$ AATTAAAAGCACGACTAACGTCTATTT- $3^{\prime}$ and $5^{\prime}$ - TTAATAGTATTCTCCACTATTGTACGATGC-3' for gallo $^{+}$. These primers amplified $502 \mathrm{bp}$ probes, which were subsequently labelled using the AlkPhos Direct Labelling and Detection system (GE Healthcare).

Assay of epimerase activity. Cells were cultured to the exponential phase in MM. A sample ( $100 \mathrm{OD}_{600}$ units) of cells was resuspended in $600 \mu \mathrm{l}$ ice-cold-buffer consisting of $0.1 \mathrm{M}$ glycine/KOH ( $\mathrm{pH} 8.7$ ) and $2 \mathrm{mM}$ EDTA. To lead to glucose repression of the cells, the cells were collected by centrifugation, and incubated for $12 \mathrm{~h}$ in MM medium supplemented with $0.1 \%$ glucose (and $2 \%$ galactose) as the carbon source. Epimerase was extracted by the glass beads method from the resuspended cells. Epimerase activity was measured by a two-step assay (Brahma \& Bhattacharyya, 2004). Briefly, extracted epimerase $(4 \mu \mathrm{l})$ and $0.5 \mathrm{mM}$ UDP-galactose $(1 \mu \mathrm{l})$ were mixed with $75 \mu \mathrm{l}$ icecold buffer. The mixture was then incubated for $30 \mathrm{~min}$ at $30^{\circ} \mathrm{C}$ and boiled for $10 \mathrm{~min}$, after which ice-cold-buffer containing $18 \mathrm{mM}$ $\mathrm{NAD}^{+}$and 12 milliunits UDP-glucose dehydrogenase (Sigma) was added. $A_{340}$ values were measured immediately for half of the sample volume (zero time) and for the other half volume after $30 \mathrm{~min}$ at $30{ }^{\circ} \mathrm{C}(30 \mathrm{~min})$. One unit of UDP-galactose 4-epimerase catalyses the 
conversion of $1 \mu \mathrm{mol}$ UDP-galactose to UDP-glucose, and thus the formation of $2 \mu \mathrm{mol} \mathrm{NADPH}$, per minute. Each nanomole of UDPgalactose converted to UDP-glucose corresponds to an $A_{340}$ change of 0.02 .

Analysis of UDP-galatose levels. Samples for analysis of UDPgalactose were prepared as described by Huang \& Snider (1995). The UDP-galactose level was measured with the Amplex Red Galactose/ Galactose Oxidase Assay kit (Invitrogen) according to the manufacturer's instructions.

Plasmid constructs. For overexpression of $\mathrm{gall}^{+}$, the cDNA was amplified by PCR using the following primers: $5^{\prime}$-GTTTTCATATGGCGGTTCAAGACGAATACA-3' (including a Nde I site) and 5'GTTTTGGATCCTTAATAGTATTCTCCACTA-3' (including a BamHI site). Derivatives of the thiamine-repressible expression vectors pREP1 and pREP41 were used to overexpress gallo (Nakamura et al., 2001).

\section{RESULTS}

\section{Sch. pombe has two types of UDP-glucose/ -galactose 4-epimerase}

Proteins that shared significant homology with human GALE and with Sac. cerevisiae GAL10 were identified through BLAST searches of the Sch. pombe genome and were designated uge $^{+} \quad(\mathrm{SPBC} 365.14 \mathrm{c})$ and gallo $^{+}$ (SPBPB2B2.12c), respectively (Fig. 1, Table 1). The Uge1 protein was characterized by the presence of only an epimerase domain. GALE and Sch. pombe Ugelp are similar in size (348 and 355 aa, respectively) and share more than $50 \%$ amino acid sequence identity (Fig. 1). On the other hand, the Gal10 protein was characterized by the presence of a mutarotase domain fused to the epimerase domain, which were also similar in size (699 and 713 aa, respectively) and also shared more than $50 \%$ amino acid sequence identity with Sc Gal10p (Fig. 1). Table 1 shows UDP-glucose/-galactose epimerase homologues from various organisms. It is interesting to note that only Schizosaccharomyces species have two types of UDPglucose/-galactose 4-epimerase, while other yeast species have only the Sc Gal10 type (epimerase and mutarotase domains). This might be related to the fact that unlike

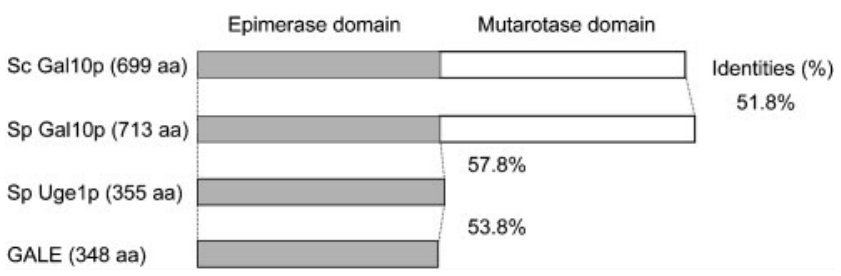

Fig. 1. Domain characterization in two types of UDP-glucose/ -galactose 4-epimerase: domain structures of the Sch. pombe Uge1 (Sp Uge1p), Sch. pombe Gal10 (Sp Gal10p), Sac. cerevisiae Gal10 (Sc Gal10p), and human GALE (GALE) proteins. Boxes representing domains with significant amino acid sequence similarity are filled in with the same pattern. other yeast species, Schizosaccharomyces species secrete galactomannoproteins and possess galactosylation mechanisms mediated by galactosyltransferases, and have UDPgalactose transporters (Chappell et al., 1994; Ohashi et al., 2009; Tabuchi et al., 1997; Umeda et al., 2000). Therefore, like mammalian cells, Schizosaccharomyces species must produce UDP-galactose constitutively from UDP-glucose via UDP-glucose/-galactose 4-epimerase as a substrate for galactosyltransferases and UDP-galactose transporters.

\section{A uge1 $\Delta$ strain exhibits a severe galactosylation defect in rich medium}

In order to determine which UDP-glucose/-galactose epimerases are functional in Sch. pombe, we constructed uge $^{+}$and $g a l 10^{+}$gene disruptants. To confirm the extent of the glycosylation defect, we examined acid phosphatase as a representative highly glycosylated glycoprotein (Dibenedetto \& Cozzani, 1975), whose migration on native gel electrophoresis depends on the size and number of $\mathrm{N}$ linked glycans (Schweingruber et al., 1986; Tanaka et al., 2001). The analysis revealed that acid phosphatase prepared from the uge $1 \Delta$ strain migrated faster than that from wild-type strains, but similarly to that from a $g m s 1 \Delta$ strain lacking the UDP-galactose transporter and which is known to contain secreted glycoproteins lacking galactose (Fig. 2a) (Tanaka et al., 2001). Acid phosphatase from the gal10 $\Delta$ strain had almost the same mobility as that from

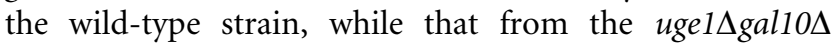
disruptant migrated similarly to acid phosphatase from the $g m s 1 \Delta$ strain. This result indicates that the oligosaccharide content is reduced in acid phosphatase prepared from the uge1s strain. To analyse the effect of cell-surface galactosylation, galactose-specific HRP-PNA staining was used to detect quantitative differences in the galactosylation of cellsurface proteins. Like the gms $1 \Delta$ mutant, neither the uge $1 \Delta$

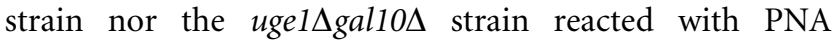
(Fig. 2b). In addition, the uge $1 \Delta$ and the uge $1 \Delta$ gallos strains exhibited sensitivity to hygromycin B (Fig. 2c), which is an aminoglycoside antibiotic used for isolating glycosylation mutants (Dean, 1995; Takegawa et al., 1996; Yoko-o et al., 2001). These results suggest that Ugelp is the predominant enzyme involved in galactosylation of glycoproteins in Sch. pombe.

\section{gal10 ${ }^{+}$is efficiently expressed in low-glucose medium but not in galactose-containing medium}

To test UDP-glucose/-galactose epimerase activity in the disruptants, the two-step assay was used (Brahma \& Bhattacharyya, 2004) (Fig. 3a). In glucose-rich medium ( $2 \%$ glucose), the uge $1 \Delta$ and the uge $1 \Delta$ gal10 $\Delta$ double mutant had about $20 \%$ activity compared to that of wildtype. On the other hand, epimerase activity of the gallos mutant showed little change. These results indicate that Ugelp is responsible for most epimerase activity in rich medium containing $2 \%$ glucose. 
Table 1. UDP-glucose/-galactose 4-epimerase homologues from various organisms

\begin{tabular}{|c|c|c|}
\hline \multirow[t]{2}{*}{ Organism } & \multicolumn{2}{|c|}{ Name of homologue (and accession number) } \\
\hline & Epimerase domain only & Epimerase + mutarotase domain \\
\hline Vanderwaltozyma polyspora DSM 70294 & - & Hypothetical protein (XP_001647142) \\
\hline Lachancea thermotolerans CBS 6340 & - & KLTH0A01958p (XP_002551545) \\
\hline Zygosaccharomyces rouxii CBS 732 & - & Hypothetical protein (XP_002494552) \\
\hline Candida dubliniensis CD36 & - & GAL10 bifunctional protein (XP_002416873) \\
\hline Candida albicans SC5314 & - & Gal10 (XP_713766) \\
\hline Debaryomyces hansenii CBS767 & - & Hypothetical protein (XP_457787) \\
\hline Pichia guilliermondii ATCC 6260 & - & Hypothetical protein (XP_001482100) \\
\hline Pichia stipitis CBS 6054 & - & Gal10 (XP_001383357) \\
\hline Yarrowia lipolytica CLIB122 & - & YALI0E26829p (XP_504440) \\
\hline Botryotinia fuckeliana B05.10 & Hypothetical protein (XP_001550259) & - \\
\hline Sclerotinia sclerotiorum 1980 & Gal10 (XP_001590586) & - \\
\hline Aspergillus niger & Hypothetical protein (XP_001401007) & - \\
\hline Aspergillus terreus NIH2624 & UDP-glucose 4-epimerase (XP_001216252) & - \\
\hline Aspergillus fumigatus Af 293 & UDP-glucose 4-epimerase (XP_753568) & - \\
\hline Aspergillus oryzae RIB40 & Hypothetical protein (XP_001827449) & - \\
\hline Talaromyces stipitatus ATCC 10500 & UDP-glucose 4-epimerase (XP_002479094) & - \\
\hline Magnaporthe grisea $70-15$ & Hypothetical protein (XP_362429) & - \\
\hline Penicillium marneffei ATCC 18224 & UDP-glucose 4-epimerase (XP_002146810) & - \\
\hline Aspergillus clavatus NRRL 1 & UDP-glucose 4-epimerase (XP_001274365) & - \\
\hline $\begin{array}{l}\text { Streptococcus gordonii strain Challis } \\
\text { substrain } \mathrm{CH} 1\end{array}$ & GalE-1 (YP_001450301) & - \\
\hline Dictyostelium discoideum AX4 & UDP-glucose 4-epimerase (XP_643834) & - \\
\hline Arabidopsis thaliana & UGE1 (NP_172738) & - \\
\hline Oryza sativa & Os09g0323000 (NP_001062869) & - \\
\hline Rattus norvegicus & GALE (NP_542961) & - \\
\hline Mus musculus & GALE (NP_848476) & - \\
\hline Homo sapiens & GALE (NP_001008217) & - \\
\hline
\end{tabular}

In Sac. cerevisiae, transcription of GAL10 is regulated by the Gal4p transcription factor, a positive regulator of gene expression for galactose-induced genes such as GAL1, GAL2, GAL7, GAL10 and MEL1, which play important roles in galactose metabolism (Hashimoto et al., 1983; Keegan et al., 1986; Lohr et al., 1995). Gal4p recognizes a 17 bp sequence ( $5^{\prime}$-CGGRNNRCYNYNCNCCG-3') in the upstream activating sequence $\left(\mathrm{UAS}_{\mathrm{GAL}}\right)$ of these genes (Lohr et al., 1995; Fedor \& Kornberg, 1989). Interestingly, the Sch. pombe genome lacks a gal4 ${ }^{+}$homologue, and the upstream sequences of $\mathrm{uge}^{+}$and $\mathrm{gallO}^{+}$also lack a typical $\mathrm{UAS}_{G A L}$ as far as we can ascertain. Therefore, we checked the mRNA levels of uge $^{+}$and gallo ${ }^{+}$in cells growing in galactose-containing medium (Fig. 3b). Northern analysis indicated the presence of $u g e 1^{+}$mRNA in cells growing in $2 \%$ glucose medium, but no increase was observed in cells grown in medium containing $2 \%$ glucose and $2 \%$ galactose. These results indicate that $u g e 1^{+}$was expressed constitutively and was not induced by galactose. On the other hand, gal10 ${ }^{+}$mRNA was expressed weakly regardless of the presence of galactose. These findings indicate that uge $^{+}$encodes a major UDP-glucose/-galactose epimerase in cells growing in rich medium containing $2 \%$ glucose. 

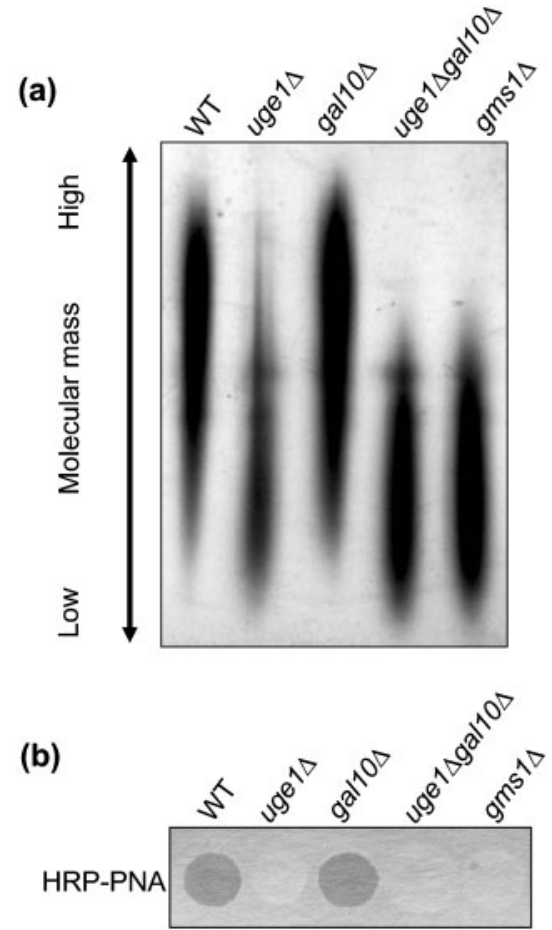

(c)
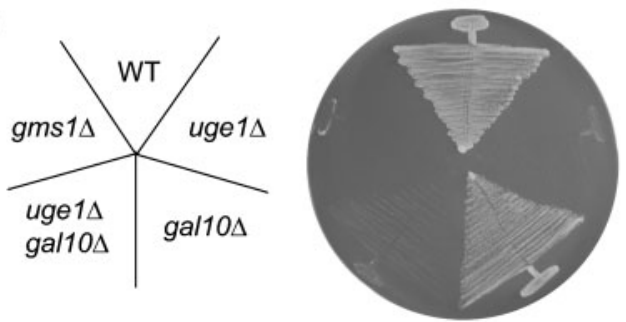

$25 \mu \mathrm{g}$ hygromycin $\mathrm{B} \mathrm{ml}^{-1}$

Fig. 2. Characterization of the glycosylation defect and growth phenotype of uge $1 \Delta$ and ga/10 $\Delta$ strains. (a) Lysates from cells induced to express acid phosphatase were subjected to gel electrophoresis and stained for acid phosphatase activity. Wildtype (WT) and each disruptant are shown. (b) The dot-spot staining assay of cell lysates indicates agglutination with HRPPNA. (c) Cells were streaked onto YES plates containing $25 \mu \mathrm{g}$ hygromycin $\mathrm{B} \mathrm{ml}^{-1}$ and incubated at $30{ }^{\circ} \mathrm{C}$ for 3 days.

In most organisms, glucose plays important regulatory roles in the expression of many genes, including those encoding proteins that function in respiration and glycolysis, utilization of alternative carbon sources, glucose transporters, and others (Johnston, 1999; Rolland et al., 2001, 2002). In Sch. pombe, however, these control mechanisms have not been analysed in detail (Tanaka et al., 1998; Hoffman, 2005; Kig et al., 2005), especially for enzymes needed for utilization of galactose as an alternative carbon source. Interestingly, Sch. pombe is not able to use galactose as a sole carbon source. Therefore, we tested whether uge $^{+}$mRNA and gal10 $0^{+}$mRNA were induced in (a)

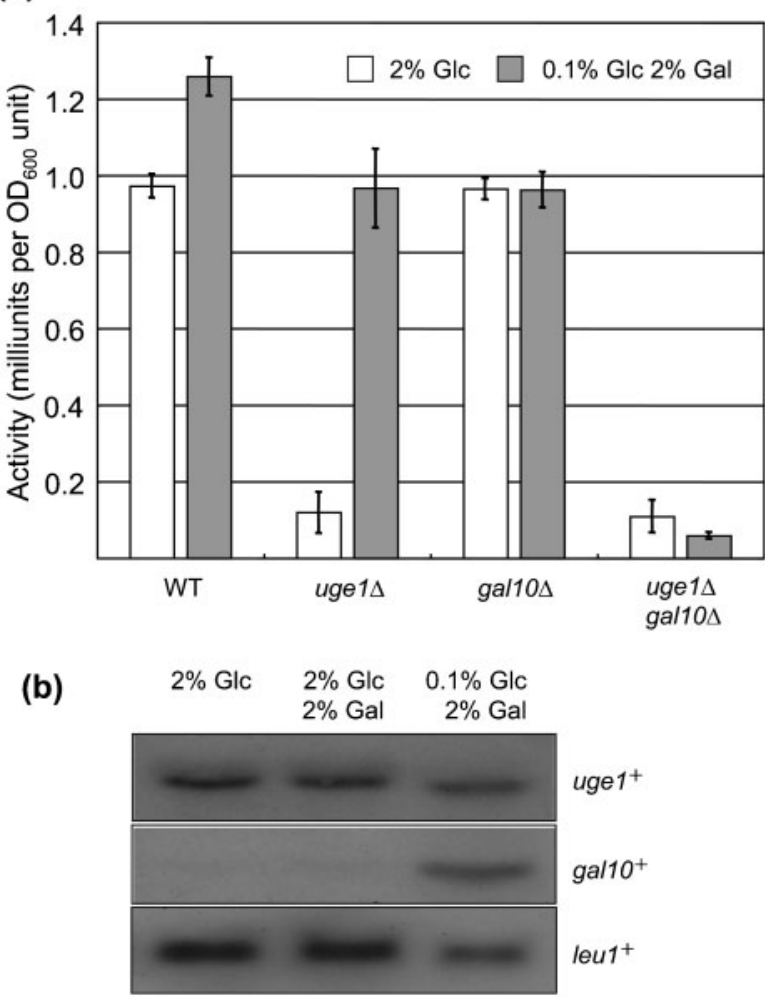

Fig. 3. Enzymic activity of UDP-glucose/-galactose 4-epimerase and expression level of uge ${ }^{+}$and ga/10 ${ }^{+}$. (a) UDP-glucose/galactose 4-epimerase activity was observed in each disruptant. Wild-type and mutant strains were cultured in MM containing $2 \%$ glucose or $0.1 \%$ glucose and $2 \%$ galactose as carbon sources. UDP-glucose 4-epimerase was extracted from the cultures and activity was monitored by measuring $A_{340}$. Values are means \pm SEM, $n=3$. (b) Northern blot analysis of $u g e 1^{+}$and gal10 $0^{+}$mRNA. Total RNA was extracted from the wild-type cells grown in glucosecontaining ( $2 \%$ glucose) medium, or in medium containing both $2 \%$ glucose and $2 \%$ galactose, or under glucose-repressing ( $0.1 \%$ glucose, $2 \%$ galactose) conditions. Each RNA sample $(20 \mu \mathrm{g})$ was separated on agarose gel in the presence of formamide, blotted onto a nylon membrane and hybridized to uge $1^{+}$, gall $10^{+}$and leu $1^{+}$probes.

glucose-repressed $(0.1 \%$ glucose, $2 \%$ galactose $)$ medium. uge $^{+}$mRNA was detected at the same level as in cells growing in $2 \%$ glucose medium, indicating that $u g e 1^{+}$ mRNA is expressed constitutively and is not induced as a consequence of glucose repression (Fig. 3b). gal10 ${ }^{+}$mRNA was detected at the same level as $u g e 1^{+}$mRNA in cells grown in $0.1 \%$ glucose, $2 \%$ galactose medium, indicating that $g a l 10^{+}$is induced during glucose repression (Fig. 3b). We next tested whether the UDP-glucose/-galactose epimerase activity in the uge1 $1 \Delta$ strain was elevated in cells grown in the $0.1 \%$ glucose $/ 2 \%$ galactose medium (Fig. $3 a$ ). The uge $1 \Delta$ strain grown in $0.1 \%$ glucose, $2 \%$ galactose medium showed a rise of UDP-glucose/-galactose epimer- 
ase activity while no detectable increase in activity was

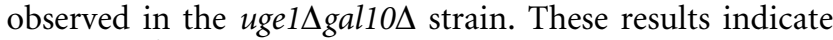
that $\mathrm{gallO}^{+}$is efficiently expressed in cells grown in $0.1 \%$ glucose medium and Gal10p has UDP-glucose/-galactose epimerase activity in Sch. pombe.

\section{Under glucose-repressing conditions, Gal10p is involved in galactosylation of cell-surface proteins}

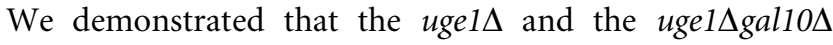
strains were not reactive with HRP-PNA and were sensitive to hygromycin B (Fig. 2b, c) due to insufficient galactosylation of secreted cell-surface glycoproteins. To verify the role of Gal10p in galactosylation, we confirmed suppression of the galactosylation defect in the uge $1 \Delta$ strain under glucose-repressing conditions (Fig. 4). The hygromycin B sensitivity of the uge $1 \Delta$ strain was overcome under preculture conditions in YES (0.1\% Glc, $2 \%$ glycerol) preculture medium, but not in YES (2\% Glc) preculture medium (Fig. 4a). Further, the HRP-PNA staining showed that galactosylation of the cell surface of the uge1 $\Delta$ mutant was restored in the YES $(0.1 \%$ Glc, $2 \%$ glycerol $)$ medium (Fig. 4b). These results indicate that Gal10p can replace loss of Ugelp in the uge $1 \Delta$ mutant in YES $(0.1 \%$ Glc, $2 \%$

(a)
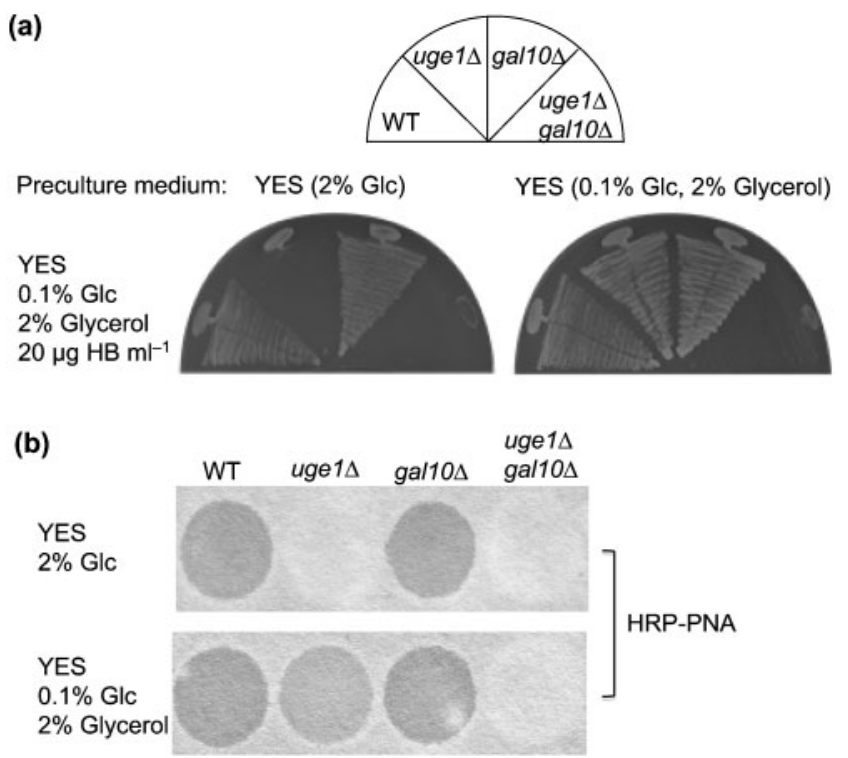

Fig. 4. Growth and galactosylation phenotypes of uge $1 \Delta$ and ga/10 $\Delta$ strains in glucose-repressing medium. (a) Wild-type and each disruptant were pre-cultured to exponential phase in YES medium containing $2 \%$ glucose or $0.1 \%$ glucose and $2 \%$ glycerol. Cells were then streaked onto YES plates $(0.1 \%$ glucose, $2 \%$ glycerol and $20 \mu \mathrm{g}$ hygromycin $\mathrm{B} \mathrm{ml} \mathrm{m}^{-1}$ ), and incubated for 3 days at $30{ }^{\circ} \mathrm{C}$. (b) The dot-spot HRP-PNA staining assay. Wildtype and each disruptant were cultured to exponential phase in YES medium containing $2 \%$ glucose or $0.1 \%$ glucose and $2 \%$ glycerol. Each cell lysate was stained with HRP-PNA. glycerol) medium, which is consistent with the activity (Fig. 3a) and expression (Fig. 3b) data.

In complementary studies, we next investigated the effect of $\mathrm{gallO}^{+}$overexpression on galactosylation in the uge $1 \Delta$ strain (Fig. 5) using the pREP1 plasmid containing the thiamine-repressible wild-type promoter (Maundrell, 1990). The induced level is about $80 \times$ greater than the repressed level. pREP41 has a $6 \times$ lower induced level and a $15 \times$ lower repressed level than the wild-type promoter (Basi et al., 1993; Maundrell, 1993; Forsburg \& Rhind, 2006). As expected, HRP-PNA staining showed that galactosylation of the cell surface of the uge1s strain was restored in an expression-dependent manner (Fig. 5a), as was the amount of acid phosphatase-associated oligosaccharides (Fig. 5b).

(a)
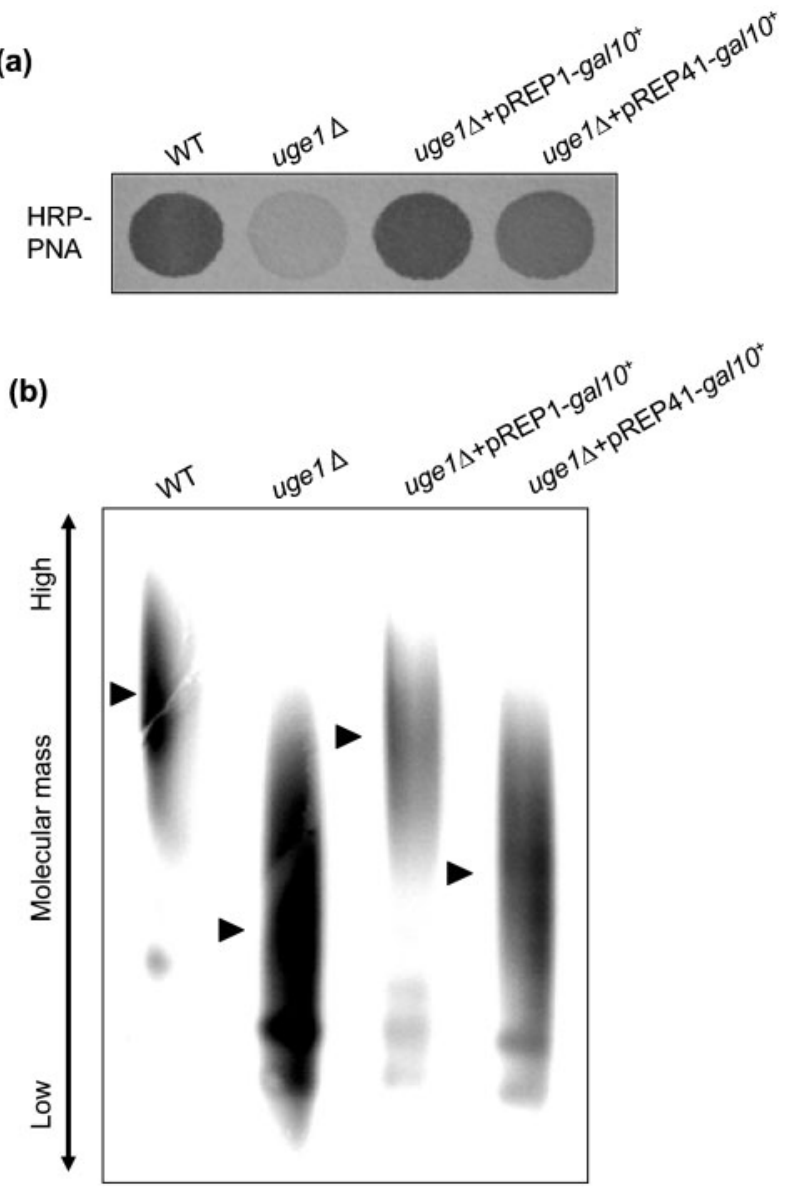

Fig. 5. Phenotype of the uge $1 \Delta$ strain overexpressing $g a / 10^{+}$ under the $n m t$ promoters. Each strain was incubated in MM containing $2 \%$ glucose for $16 \mathrm{~h}$. The uge $1 \Delta$ strain overexpressed gal $10^{+}$either under the pREP1 or pREP41 $\mathrm{nmt}$ promoters. Cell lysates of each strain were stained with HRP-PNA (a), and subjected to gel electrophoresis followed by staining for acid phosphatase activity (b). 


\section{Gal7p is associated with UDP-galactose biosynthesis in Sch. pombe}

As documented above, Sch. pombe cannot grow on galactose-containing medium with galactose as sole carbon source. This phenotype has greatly complicated analysis of the Leloir pathway in this yeast. Therefore, we wanted to determine whether galactose was transported from the medium to the cytosol and whether it was converted into UDP-galactose via Gal-1-P. The uge1sgallos strain is useful for assessing this pathway in Sch. pombe. If galactosylation of the cell surface is recovered on galactose-containing medium in the uge1 1 gallos strain, this indicates that the galactose residues in the glycoproteins were synthesized using UDP-galactose formed from Gal-1-P by Gal-1-P uridylyltransferase. Fig. 6(a) shows that HRP-PNA staining of the uge1s and uge $1 \Delta$ gallos strains was indeed restored in the galactose-containing medium.

In Sch. pombe, a search for homologue(s) of Sac. cerevisiae GAL7, which encodes Gal-1-P uridylyltransferase, identified SPBPB2B2.10c, designated gal $^{+}$in this study. The predicted amino acid sequences of the Sc GAL7 and $g a l 7^{+}$

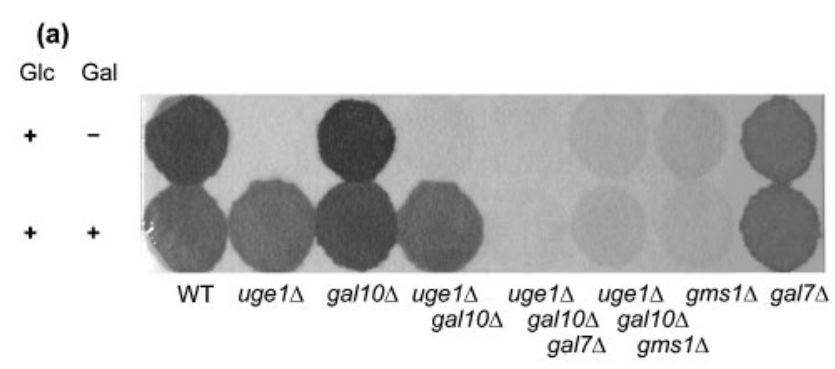

(b)

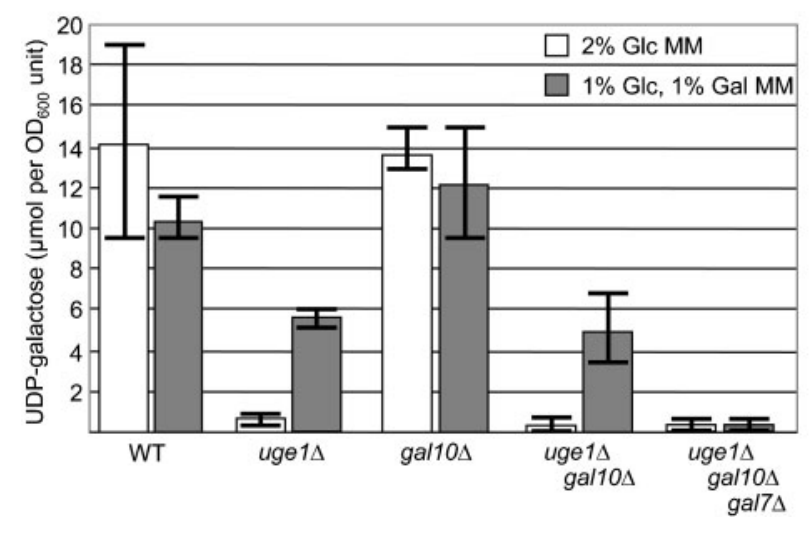

Fig. 6. Galactosylation phenotype and UDP-galactose content in cells grown in galactose-containing medium. (a) Cells were incubated in $\mathrm{MM}$ containing $2 \%$ glucose $(\mathrm{Glc}+, \mathrm{Gal}-)$, or $1 \%$ glucose and $1 \%$ galactose $(\mathrm{Glc}+, \mathrm{Gal}+)$. Cell lysates of each strain were stained with HRP-PNA. (b) UDP-galactose was extracted from strains cultured to exponential phase in $\mathrm{MM}$ containing $2 \%$ glucose ( $2 \%$ Glc MM) or $1 \%$ glucose and $1 \%$ galactose (1\% Glc, $1 \%$ Gal MM). UDP-galactose content was monitored by measuring $A_{340}$. Values are means $\pm \mathrm{SEM}, n=3$. gene products share $63 \%$ identity. To examine whether $\mathrm{gall}^{+}$is involved in the recovery of HRP-PNA staining, we

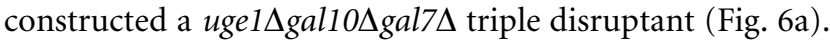
HRP-PNA staining was not observed in either the

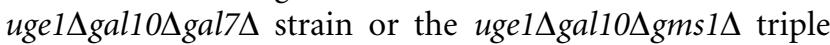
disruptant in galactose-containing medium. These results indicate that galactose is transported into the cytosol from the medium and is converted into UDP-galactose via Gal1-P by Gal7p in Sch. pombe.

We next determined the UDP-galactose content of the cytosol in these strains (Fig. 6b). All the disruptants except gal10 $\Delta$ had reduced levels of cytosolic UDP-galactose when grown in $2 \%$ glucose medium. After incubation in medium containing $1 \%$ galactose, the uge $1 \Delta$ and uge $1 \Delta$ gal10 $\Delta$ strains had increased amounts of cytosolic UDP-galactose. In contrast, increased UDP-galactose content in the uge $1 \Delta$ gal10 $\Delta$ gal7 $\Delta$ strain was not detected in cells grown in the $1 \%$ galactose-containing medium. These results indicate that Gal7p is involved in UDPgalactose biosynthesis in Sch. pombe.

\section{DISCUSSION}

Glycoproteins in Sch. pombe contain a large amount of galactose in addition to both $N$-linked and $O$-linked mannan (Manners \& Meyer, 1977; Moreno et al., 1985; Ikeda et al., 2009; Ohashi et al., 2009). The galactose residues are modified by galactosyltransferases using UDPgalactose as substrate (Andreishcheva et al., 2004; Chappell et al., 1994; Yoko-o et al., 1998). UDP-galactose is transported to the Golgi lumen from the cytosol by a Golgi-localized UDP-galactose transporter (Gmslp) (Tabuchi et al., 1997; Tanaka \& Takegawa, 2001). In Sch. pombe, biosynthesis of cytosolic UDP-galactose is poorly characterized. UDP-galactose/-glucose 4-epimerase is the key enzyme involved in UDP-galactose synthesis in the cytosol, where it catalyses the interconversion of UDPgalactose and UDP-D-glucose. Interestingly, Schizosaccharomyces species have two types of UDP-glucose/-galactose 4-epimerase homologues, although other organisms have only one kind. It is not known why two types of epimerase are needed and how they are regulated in Sch. pombe.

Here we show (1) that Sch. pombe has two genes encoding functional epimerases (named $u g e 1^{+}$and gal10 ${ }^{+}$); (2) that Ugelp is a major UDP-glucose/-galactose epimerase expressed constitutively in cells grown in glucose-rich medium ( $2 \%$ glucose, Fig. $7 \mathrm{a})$; (3) that gal10 ${ }^{+}$is efficiently expressed and involved in galactosylation of cell surface proteins in low-glucose medium $(0.1 \%$ glucose, Fig. $7 \mathrm{a})$, but not in galactose-containing medium; and (4) that galactose is transported into the cytosol and is converted into UDP-galactose via Gal-1-P by Gal7p in Sch. pombe (Fig. 7b).

In this study, we shed new light on the Leloir pathway from the standpoint of UDP-galactose synthesis in Sch. pombe (Fig. 7). It is interesting to note that Schizosaccharomyces 

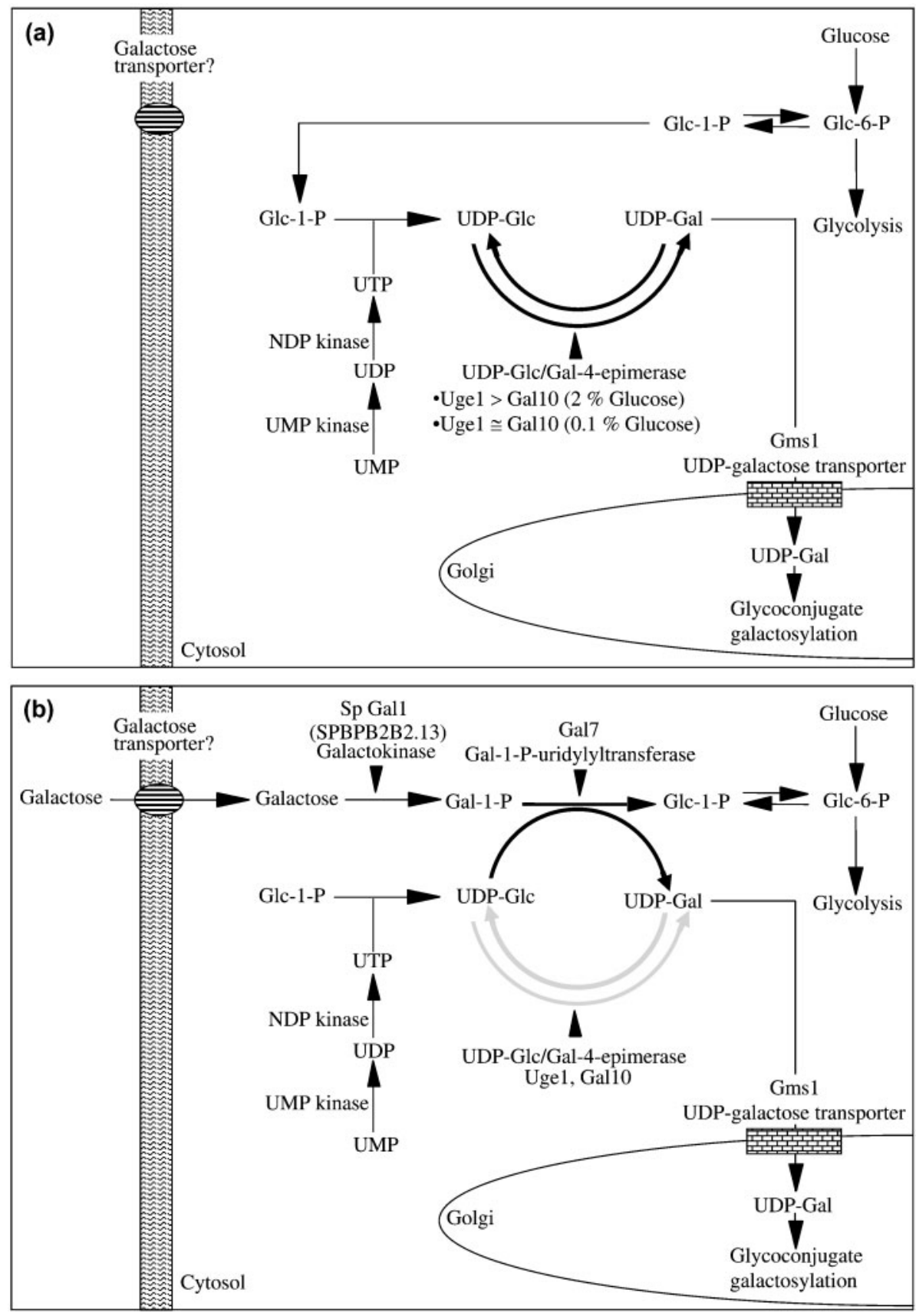

Fig. 7. Proposed model for UDP-galactose synthesis in Sch. pombe. (a) UDP-galactose synthesis in glucose medium. Uge1p is constitutively expressed in $2 \%$ glucose medium (Uge1>Gal10) and Gal10p is induced to the same level as Uge1p

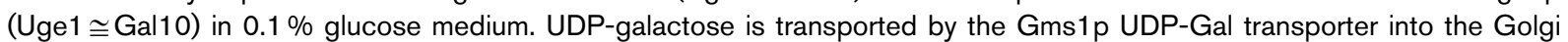
lumen, where it is utilized for galactosylation of glycoproteins. (b) UDP-galactose synthesis in the uge $1 \Delta$ gal $10 \Delta$ strain in galactose-containing medium. Galactose is transported into the cytosol from the medium by unidentified transporters and is converted into UDP-galactose via galactose-1-phosphate by Gal7p.

species are the only organisms known to have two types of UDP-glucose/-galactose 4-epimerase (Figs 1 and 7, Table 1). To our knowledge, other yeast species only have the Sc Gal10 type of epimerase, which has both epimerase and mutarotase domains. A key difference between these yeast species and
Sch. pombe is that Sch. pombe can modify galactose for use in synthesizing glycoproteins, while other yeasts modify mannose for the same purpose (Gemmill \& Trimble, 1999). This might suggest that the Uge1 type of epimerase with only an epimerase domain could have evolved for 
efficient UDP-galactose production for galactosylation of glycoproteins in Sch. pombe. We previously reported that disruption of the $g m s 1^{+}$gene led to a complete loss of protein galactosylation, due to a defect in transport of UDPgalactose from the cytosol to the lumen of the Golgi apparatus as a substrate for galactosyltransferase (Fig. 7) (Tabuchi et al., 1997; Tanaka et al., 2001). The gms1D strain exhibited aberrant cell morphology and increased sensitivity to digestion with $\beta$-glucanase and to various drugs, such as hygromycin B, sodium orthovanadate and calcofluor white. Further, the gms1s strain was incapable of sexual conjugation during nutritional starvation and of non-sexual flocculation (Tanaka et al., 1999, 2001). The uge1s strain also had a severe galactosylation defect and a decrease in epimerase activity and UDP-galactose content when grown in glucose-rich medium ( $2 \%$ glucose) (Figs 2,3 and $6 \mathrm{~b}$ ), indicating that Ugelp is a major UDP-glucose/-galactose epimerase expressed constitutively in $2 \%$ glucose medium (Fig. 7a).

In Sac. cerevisiae, all the galactose-inducible genes (GAL1, GAL10, GAL7 and GAL2) are coordinately regulated at the level of transcription in response to galactose by Gal4p, Gal80p and Gal3p (De Robichon-Szulmajster, 1958; Lohr et al., 1995; Platt \& Reece, 1998). In the absence of galactose, binding of Gal80p to Gal4p effectively limits active transcription of the GAL genes. In the presence of galactose, Gal3p-Gal80p interactions are triggered in the cytoplasm, resulting in redistribution of Gal80p from the nucleus to the cytoplasm, thereby relieving inhibition of Gal4p and resulting in GAL gene expression (Peng \& Hopper, 2002). However, the Sch. pombe genome has no homologues of Gal4p, Gal80p or Gal3p. Further, the promoter sequences of $\mathrm{ugel}^{+}, \mathrm{gallo}^{+}$and $\mathrm{gall}^{+}$also lack typical Gal4p-binding sequences [UAS ${ }_{G A L}: 5^{\prime}$-CGGRNNRCYNYNCNCCG-3' (Fedor \& Kornberg, 1989; Lohr et al., 1995)]. In this study, we found that gal10 ${ }^{+}$is efficiently expressed and involved in galactosylation of cell-surface proteins in cells grown in low $(0.1 \%)$ glucose medium, but not in galactose-containing medium (Figs $3 \mathrm{~b}, 4$ and $7 \mathrm{a}$ ). We previously reported that the $i n v 1^{+}$gene that encodes invertase is repressed in the presence of glucose (Tanaka et al., 1998). The transcription of $i n v 1^{+}$is regulated by Scrlp, which is required for glucose repression of $i n v 1^{+}$ (Tanaka et al., 1998) and $f b p 1^{+}$(Neely \& Hoffman, 2000). Similarly, Scrlp may have repressed the transcription of

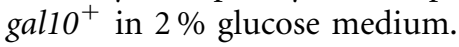

Sch. pombe is not able to grow on galactose-containing medium with galactose as sole carbon source. This phenotype makes it difficult to analyse the Leloir pathway in Sch. pombe, despite the presence of the three relevant enzymes, galactokinase, Gal-1-P uridylyltransferase and UDP-glucose/-galactose epimerase. To address this problem, we used a uge $1 \Delta$ gal10 $\Delta$ strain. Recovery of galacto-

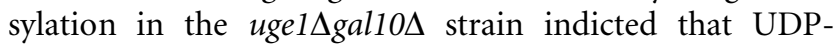
galactose was produced by sequential reaction of galactokinase and Gal7 without UDP-glucose/-galactose epimerase (Figs 6 and $7 \mathrm{~b}$ ). These results also suggest that Sch. pombe is able to transport galactose from the medium into the cytosol by unidentified transporters. In Sac. cerevisiae, Gal2p (Tschopp et al., 1986), which is an integral plasma membrane protein predicted to contain 12 transmembrane domains (Nehlin et al., 1989) is able to transport galactose. GAL2 is also regulated at the level of transcription in response to galactose by Gal4p (De Robichon-Szulmajster, 1958; Lohr et al., 1995; Platt \& Reece, 1998), and loss of Gal2p's permease activity renders cells unable to utilize galactose as a sole carbon source (Douglas \& Condie, 1954). In contrast, a GAL2 homologue was not found in the Sch. pombe genome. Multiple genes that encode hexose transporters (Ght1 to Ght6) have been identified in Sch. pombe (Heiland et al., 2000). These transporters are involved in glucose, fructose and gluconate transport from the extracellular milieu into the cytosol (Heiland et al., 2000). However, a galactose transporter has not yet been

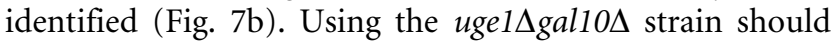
help reveal not only the function of galactokinase and Gal7p, but also the galactose transport machinery in Sch. pombe. Schizosaccharomyces japonicus and Sch. octosporus also lack genes involved in regulation of the Leloir pathway, suggesting that the features of galactose metabolism identified in Sch. pombe may also apply in other Schizosaccharomyces species.

We were not able to analyse the mutarotase domain of Gal10p in this study. Gal10p was efficiently expressed under low $(0.1 \%)$ glucose conditions, suggesting that mutarotase activity might be elevated under these conditions. Further studies will be necessary to determine how galactokinase and Gal7p are regulated under various growth conditions in Sch. pombe.

\section{ACKNOWLEDGEMENTS}

We thank Drs Taro Nakamura and Yuko Giga-Hama for providing Sch. pombe plasmids and strains. This work was supported in part by a Grant-in-Aid for Scientific Research from the Ministry of Education, Science, and Culture of Japan.

\section{REFERENCES}

Alfa, C., Fantes, P., Hyams, J., McLoed, M. \& Warbrick, E. (1993). Experiments with Fission Yeast: a Laboratory Course Manual. Cold Spring Harbor, NY: Cold Spring Harbor Laboratory.

Andreishcheva, E. N., Kunkel, J. P., Gemmill, T. R. \& Trimble, R. B. (2004). Five genes involved in biosynthesis of the pyruvylated $\mathrm{Gal} \beta$ 1,3-epitope in Schizosaccharomyces pombe $N$-linked glycans. J Biol Chem 279, 35644-35655.

Basi, G., Schmid, E. \& Maundrell, K. (1993). TATA box mutations in the Schizosaccharomyces pombe nmt1 promoter affect transcription efficiency but not the transcription start point or thiamine repressibility. Gene 123, 131-136.

Brahma, A. \& Bhattacharyya, D. (2004). UDP-galactose 4-epimerase from Kluyveromyces fragilis. Evidence for independent mutarotation site. Eur J Biochem 271, 58-68.

Chappell, T. G., Hajibagheri, M. A., Ayscough, K., Pierce, M. \& Warren, G. (1994). Localization of an $\alpha-1,2$ galactosyltransferase 
activity to the Golgi apparatus of Schizosaccharomyces pombe. Mol Biol Cell 5, 519-528.

Dean, N. (1995). Yeast glycosylation mutants are sensitive to aminoglycosides. Proc Natl Acad Sci U S A 92, 1287-1291.

De Robichon-Szulmajster, H. (1958). Induction of enzymes of the galactose pathway in mutants of Saccharomyces cerevisiae. Science 127, 28-29.

Dibenedetto, G. \& Cozzani, I. (1975). Nonspecific acid phosphatase from Schizosaccharomyces pombe. Purification and physical chemical properties. Biochemistry 14, 2847-2852.

Douglas, H. C. \& Condie, F. (1954). The genetic control of galactose utilization in Saccharomyces. J Bacteriol 68, 662-670.

Fedor, M. J. \& Kornberg, R. D. (1989). Upstream activation sequencedependent alteration of chromatin structure and transcription activation of the yeast GAL1-GAL10 genes. Mol Cell Biol 9, 1721-1732.

Forsburg, S. L. \& Rhind, N. (2006). Basic methods for fission yeast. Yeast 23, 173-183.

Frey, P. A. (1996). The Leloir pathway: a mechanistic imperative for three enzymes to change the stereochemical configuration of a single carbon in galactose. FASEB J 10, 461-470.

Gemmill, T. R. \& Trimble, R. B. (1996). Schizosaccharomyces pombe produces novel pyruvate-containing $\mathrm{N}$-linked oligosaccharides. J Biol Chem 271, 25945-25949.

Gemmill, T. R. \& Trimble, R. B. (1998). All pyruvylated galactose in Schizosaccharomyces pombe $N$-glycans is present in the terminal disaccharide, $\quad 4,6-O-[(R)$-(1-carboxyethylidine $)]-G a l \beta \quad 1,3 \mathrm{Gal} \alpha 1$. Glycobiology 8, 1087-1095.

Gemmill, T. R. \& Trimble, R. B. (1999). Overview of $N$ - and $O$-linked oligosaccharide structures found in various yeast species. Biochim Biophys Acta 1426, 227-237.

Hashimoto, H., Kikuchi, Y., Nogi, Y. \& Fukasawa, T. (1983). Regulation of expression of the galactose gene cluster in Saccharomyces cerevisiae. Isolation and characterization of the regulatory gene GAL4. Mol Gen Genet 191, 31-38.

Heiland, S., Radovanovic, N., Hofer, M., Winderickx, J. \& Lichtenberg, H. (2000). Multiple hexose transporters of Schizosaccharomyces pombe. J Bacteriol 182, 2153-2162.

Hoffman, C. S. (2005). Except in every detail: comparing and contrasting G-protein signaling in Saccharomyces cerevisiae and Schizosaccharomyces pombe. Eukaryot Cell 4, 495-503.

Holden, H. M., Rayment, I. \& Thoden, J. B. (2003). Structure and function of enzymes of the Leloir pathway for galactose metabolism. J Biol Chem 278, 43885-43888.

Huang, K. M. \& Snider, M. D. (1995). Isolation of protein glycosylation mutants in the fission yeast Schizosaccharomyces pombe. Mol Biol Cell 6, 485-496.

Ikeda, Y., Ohashi, T., Tanaka, N. \& Takegawa, K. (2009). Identification and characterization of a gene required for $\alpha 1,2$ mannose extension in the $O$-linked glycan synthesis pathway in Schizosaccharomyces pombe. FEMS Yeast Res 9, 115-125.

Johnston, M. (1999). Feasting, fasting and fermenting. Glucose sensing in yeast and other cells. Trends Genet 15, 29-33.

Keegan, L., Gill, G. \& Ptashne, M. (1986). Separation of DNA binding from the transcription-activating function of a eukaryotic regulatory protein. Science 231, 699-704.

Kig, C., Turkel, S. \& Temizkan, G. (2005). Isolation and characterization of glucose derepressed invertase mutants from Schizosaccharomyces pombe. Biosci Biotechnol Biochem 69, 2475-2478.

Lohr, D., Venkov, P. \& Zlatanova, J. (1995). Transcriptional regulation in the yeast $G A L$ gene family: a complex genetic network. FASEB J $\mathbf{9}$ 777-787.
Majumdar, S., Ghatak, J., Mukherji, S., Bhattacharjee, H. \& Bhaduri, A. (2004). UDPgalactose 4-epimerase from Saccharomyces cerevisiae. A bifunctional enzyme with aldose 1-epimerase activity. Eur J Biochem 271, 753-759.

Manners, D. J. \& Meyer, M. T. (1977). The molecular structures of some glucans from the cell walls of Schizosaccharomyces pombe. Carbohydr Res 57, 189-203.

Maundrell, K. (1990). $n m t 1$ of fission yeast. A highly transcribed gene completely repressed by thiamine. J Biol Chem 265, 10857-10864.

Maundrell, K. (1993). Thiamine-repressible expression vectors pREP and pRIP for fission yeast. Gene 123, 127-130.

Moreno, S., Ruiz, T., Sanchez, Y., Villanueva, J. R. \& Rodriguez, L. (1985). Subcellular localization and glycoprotein nature of the invertase from the fission yeast Schizosaccharomyces pombe. Arch Microbiol 142, 370-374.

Moreno, S., Klar, A. \& Nurse, P. (1991). Molecular genetic analysis of fission yeast Schizosaccharomyces pombe. Methods Enzymol 194, 795823.

Morita, T. \& Takegawa, K. (2004). A simple and efficient procedure for transformation of Schizosaccharomyces pombe. Yeast 21, 613-617.

Nakamura, T., Nakamura-Kubo, M., Hirata, A. \& Shimoda, C. (2001). The Schizosaccharomyces pombe spo $3^{+}$gene is required for assembly of the forespore membrane and genetically interacts with $p s y 1^{+}$encoding syntaxin-like protein. Mol Biol Cell 12, 3955-3972.

Neely, L. A. \& Hoffman, C. S. (2000). Protein kinase A and mitogenactivated protein kinase pathways antagonistically regulate fission yeast $f b p 1$ transcription by employing different modes of action at two upstream activation sites. Mol Cell Biol 20, 6426-6434.

Nehlin, J. O., Carlberg, M. \& Ronne, H. (1989). Yeast galactose permease is related to yeast and mammalian glucose transporters. Gene 85, 313-319.

Ohashi, T., Ikeda, Y., Tanaka, N., Nakakita, S., Natsuka, S., Giga-Hama, Y. \& Takegawa, K. (2009). The och1 mutant of Schizosaccharomyces pombe produces galactosylated core structures of N-linked oligosaccharides. Biosci Biotechnol Biochem 73, 407-414.

Okazaki, K., Okazaki, N., Kume, K., Jinno, S., Tanaka, K. \& Okayama, H. (1990). High-frequency transformation method and library transducing vectors for cloning mammalian cDNAs by transcomplementation of Schizosaccharomyces pombe. Nucleic Acids Res 18, 6485-6489.

Peng, G. \& Hopper, J. E. (2002). Gene activation by interaction of an inhibitor with a cytoplasmic signaling protein. Proc Natl Acad Sci U S A 99, 8548-8553.

Platt, A. \& Reece, R. J. (1998). The yeast galactose genetic switch is mediated by the formation of a Gal4p-Gal80p-Gal3p complex. EMBO J 17, 4086-4091.

Rolland, F., Winderickx, J. \& Thevelein, J. M. (2001). Glucose-sensing mechanisms in eukaryotic cells. Trends Biochem Sci 26, 310-317.

Rolland, F., Winderickx, J. \& Thevelein, J. M. (2002). Glucose-sensing and -signalling mechanisms in yeast. FEMS Yeast Res 2, 183-201.

Sambrook, J., Fritsch, E. \& Maniatis, T. (1989). Molecular Cloning: a Laboratory Manual, 2nd edn. Cold Spring Harbor, NY: Cold Spring Harbor Laboratory.

Schweingruber, M. E., Fluri, R., Maundrell, K., Schweingruber, A. M. \& Dumermuth, E. (1986). Identification and characterization of thiamin repressible acid phosphatase in yeast. J Biol Chem 261, 15877-15882.

Scott, A. \& Timson, D. J. (2007). Characterization of the Saccharomyces cerevisiae galactose mutarotase/UDP-galactose 4-epimerase protein, Gal10p. FEMS Yeast Res 7, 366-371. 
Suga, M. \& Hatakeyama, T. (2001). High efficiency transformation of Schizosaccharomyces pombe pretreated with thiol compounds by electroporation. Yeast 18, 1015-1021.

Suga, M., Isobe, M. \& Hatakeyama, T. (2000). Cryopreservation of competent intact yeast cells for efficient electroporation. Yeast 16, 889-896.

Tabuchi, M., Tanaka, N., Iwahara, S. \& Takegawa, K. (1997). The Schizosaccharomyces pombe $g m s 1^{+}$gene encodes an UDP-galactose transporter homologue required for protein galactosylation. Biochem Biophys Res Commun 232, 121-125.

Takegawa, K., Tanaka, N., Tabuchi, M. \& Iwahara, S. (1996). Isolation and characterization of a glycosylation mutant from Schizosaccharomyces pombe. Biosci Biotechnol Biochem 60, 1156-1159.

Tanaka, N. \& Takegawa, K. (2001). Functional characterization of Gms1p/UDP-galactose transporter in Schizosaccharomyces pombe. Yeast 18, 745-757.

Tanaka, N., Ohuchi, N., Mukai, Y., Osaka, Y., Ohtani, Y., Tabuchi, M., Bhuiyan, M. S., Fukui, H., Harashima, S. \& Takegawa, K. (1998). Isolation and characterization of an invertase and its repressor genes from Schizosaccharomyces pombe. Biochem Biophys Res Commun 245, 246-253.

Tanaka, N., Awai, A., Bhuiyan, M. S., Fujita, K., Fukui, H. \& Takegawa, K. (1999). Cell surface galactosylation is essential for nonsexual flocculation in Schizosaccharomyces pombe. J Bacteriol 181, 1356-1359.
Tanaka, N., Konomi, M., Osumi, M. \& Takegawa, K. (2001). Characterization of a Schizosaccharomyces pombe mutant deficient in UDP-galactose transport activity. Yeast 18, 903-914.

Thoden, J. B. \& Holden, H. M. (2005). The molecular architecture of galactose mutarotase/UDP-galactose 4-epimerase from Saccharomyces cerevisiae. J Biol Chem 280, 21900-21907.

Tschopp, J. F., Emr, S. D., Field, C. \& Schekman, R. (1986). GAL2 codes for a membrane-bound subunit of the galactose permease in Saccharomyces cerevisiae. J Bacteriol 166, 313-318.

Umeda, K., Yoko-o, T., Nakayama, K., Suzuki, T. \& Jigami, Y. (2000). Schizosaccharomyces pombe gmd $3^{+} /$alg $11^{+}$is a functional homologue of Saccharomyces cerevisiae ALG11 which is involved in $N$-linked oligosaccharide synthesis. Yeast 16, 1261-1271.

Yoko-o, T., Roy, S. K. \& Jigami, Y. (1998). Differences in in vivo acceptor specificity of two galactosyltransferases, the $g m h 3^{+}$and

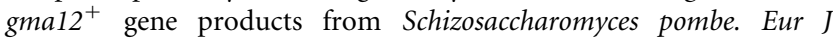
Biochem 257, 630-637.

Yoko-o, T., Tsukahara, K., Watanabe, T., Hata-Sugi, N., Yoshimatsu, K., Nagasu, T. \& Jigami, Y. (2001). Schizosaccharomyces pombe och $1^{+}$ encodes $\alpha$-1,6-mannosyltransferase that is involved in outer chain elongation of $N$-linked oligosaccharides. FEBS Lett 489, 75-80.

Edited by: M. Schweizer 